\title{
Modulation characteristics of tunable DFB/DBR lasers with one or two passive tuning sections
}

Pan, Xing; Olesen, Henning; Tromborg, Bjarne

Published in:

I E E E Journal of Quantum Electronics

Link to article, DOI:

10.1109/3.29256

Publication date:

1989

Document Version

Publisher's PDF, also known as Version of record

Link back to DTU Orbit

Citation (APA):

Pan, X., Olesen, H., \& Tromborg, B. (1989). Modulation characteristics of tunable DFB/DBR lasers with one or two passive tuning sections. I E E E Journal of Quantum Electronics, 25(6), 1254-1260.

https://doi.org/10.1109/3.29256

\section{General rights}

Copyright and moral rights for the publications made accessible in the public portal are retained by the authors and/or other copyright owners and it is a condition of accessing publications that users recognise and abide by the legal requirements associated with these rights.

- Users may download and print one copy of any publication from the public portal for the purpose of private study or research.

- You may not further distribute the material or use it for any profit-making activity or commercial gain

- You may freely distribute the URL identifying the publication in the public portal 


\title{
Modulation Characteristics of Tunable DFB/DBR Lasers with One or Two Passive Tuning Sections
}

\author{
XING PAN, HENNING OLESEN, AND BJARNE TROMBORG
}

\begin{abstract}
Calculation of the small-signal modulation characteristics of tunable DFB and DBR lasers requires rate equations for the photon number, the phase of the electric field, and the carrier densities. In this paper, we derive the rate equation for the photon number and phase from an optical transmission line model and present examples of the frequency and intensity modulation characteristics of a phasetunable DFB laser. The modulation responses exhibit the well-known relaxation resonance if either of the drive currents is modulated, but we also demonstrate the possibility of complete removal of the resonance peak together with a perfect cancellation of spurious intensity modulation. The frequency modulation response then assumes a simple low-pass character with a cutoff frequency determined by the carrier lifetime in the passive tuning section. The importance of choosing the proper bias conditions is emphasized.
\end{abstract}

\section{INTRODUCTION}

A LARGE variety of tunable multisection DFB and DBR lasers is currently under investigation, and several experimental results have been reported. These lasers typically consist of two to four sections with separate electrodes, and depending on the application, all sections may be active (in the simplest form just a conventional DFB laser with segmented electrodes) [1], one section may act as a saturable absorber in order to achieve bistable operation or optical wavelength conversion [2], or the device may have one active (Fabry-Perot- or DFB-type) and one or two passive sections [3]-[6]. The main advantages of such devices are the capability of fast electronic tuning, a low sensitivity to environmental disturbances, and for the latter type, a large continuous tuning range.

In this paper, we will focus on devices with passive tuning sections. In addition to the advantages mentioned above, these lasers are attractive because of the possibility of getting a high frequency modulation (FM) response, which is uniform in modulation frequency from dc to several hundred megahertz, by modulation of the injection current to one of the passive sections [3]. The carrier density in the passive sections is not clamped as it is in a conventional laser above threshold and is therefore easy

Manuscript received October 6. 1988; revised Janury 18, 1989. This work was supported by the EEC under Contract ST2J-0171-2-DK.

$\mathrm{X}$. Pan is with the Electromagnetics Institute, Technical University of Denmark, DK-2800 Lyngby, Denmark.

$\mathrm{H}$. Olesen and B. Tromborg are with the TFL Telecommunications Research Laboratory, DK-2970 Horsholm, Denmark.

IEEE Log Number 8927324 to modulate. The cutoff frequency will be determined by the carrier lifetime in the passive section. We have previously analyzed the static tuning properties of DFB and DBR lasers with passive tuning sections [7], [8], and in this paper, which is an extended version of [9], we present the first theoretical study of their modulation characteristics.

\section{THEORY}

The analysis presented here is an application of our general transmission line theory for compound cavity lasers [10]. It applies to electronically tunable devices with one active and one or two passive control sections. The most important devices in this category are the phase-tunable DFB laser [3] with one passive phase control (PC) section and the three-section tunable DBR laser [4], which has an active Fabry-Perot section and two passive sections, one for phase control and one with a built-in corrugation for frequency control. The passive waveguides are usually made of materials with a higher bandgap than that of the active layer in order to make them transparent (apart from the intrinsic absorption). Hence, there will be only spontaneous and no stimulated recombination in these sections. In the following, we will limit the discussion to the phase-tunable DFB laser, which is shown schematically in Fig. 1, but the modifications for the DBR laser are straightforward.

Initially, we introduce a reference plane at the interface between the active and PC sections. The right and left traveling waves $E^{ \pm}(\omega)$ at the reference plane are related by the boundary conditions

$$
\begin{aligned}
& E^{+}(\omega)=r_{L}\left(\omega, N_{1}\right) E^{-}(\omega)+F_{L}(\omega) \\
& E^{-}(\omega)=r_{R}\left(\omega, N_{2}\right) E^{+}(\omega)
\end{aligned}
$$

where $r_{L}$ and $r_{R}$ are the effective reflectivities "seen" towards the left and right from the reference plane, $\omega$ is the angular optical frequency, and $N_{1}$ and $N_{2}$ are the carrier densities of the active and the $\mathrm{PC}$ sections, respectively. $F_{L}$ is a Langevin noise term, which accounts for the spontaneous emission in the active section [10]. There is no contribution from spontaneous emission in the PC section because of the higher bandgap.

Expressions for $r_{L}$ and $r_{R}$ are given in [7, eqs. (4) and (12)]. $r_{L}$ is derived from the well-known coupled mode

0018-9197/89/0600-1254\$01.00 (C) 1989 IEEE 


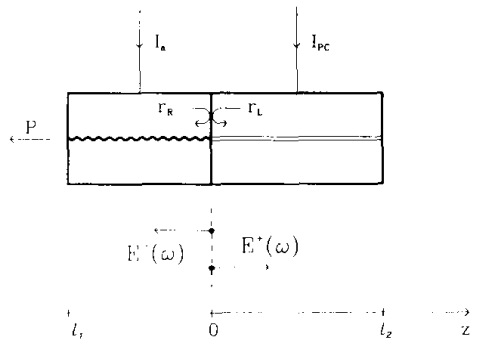

Fig. 1. Phase tunable DFB laser with an active DFB section and a passive $\mathrm{PC}$ section

equations for DFB lasers [11], and if the reflections at the laser-waveguide interface are neglected, $r_{R}$ takes the simple form

$$
r_{R}\left(\omega, N_{2}\right)=r_{2} \exp \left\{-j 2 k_{2}\left(\omega, N_{2}\right) l_{2}\right\}
$$

where $r_{2}$ is the facet reflectivity at the end of the PC section, $k_{2}\left(\omega, N_{2}\right)$ is the complex wave number, and $l_{2}$ is the length of the PC section. Recent experimental results indicate that the residual reflections at the interfaces can indeed be negligible for butt-jointed devices fabricated by the MOVPE technique [6], [12]. Equation (2) shows that $r_{R}$ depends implicitly on $\omega$ and $N_{2}$ through the complex wave number $k_{2}\left(\omega, N_{2}\right)$. Similarly, $r_{L}$ depends on $\omega$ and $N_{1}$ through the complex wave number $k_{1}\left(\omega, N_{1}\right)$ for the active section. The expressions for $k_{1}$ and $k_{2}$ are given in Appendix A.

Equations (la) and (1b) can be combined to give the basic field equation

$$
\left\{r_{L}\left(\omega, N_{1}\right) r_{R}\left(\omega, N_{2}\right)-1\right\} E^{+}(\omega)=F_{L}(\omega) .
$$

The driving term $F_{L}(\omega)$ is discussed in detail in [10]. In the present analysis of modulation properties, we shall simply ignore the term and put $F_{L}(\omega)$ equal to 0 .

The stationary operation point $\left(\omega_{s}, N_{1 s}, N_{2 s}\right)$ (without noise or modulation) satisfies the oscillation condition

$$
r_{L}\left(\omega_{s}, N_{l s}\right) r_{R}\left(\omega_{s}, N_{2 s}\right)=1 .
$$

The dominant mode is the particular solution to (4), which has the lowest threshold carrier density $N_{1 s}$. The carrier density $N_{2 s}$ is independently determined by the injection current to the PC section and the condition that the carrier injection rate equals the spontaneous recombination rate [7]. The method for solving (4) is described in [7].

Under dynamic conditions, the frequency and carrier densities are assumed to be close to their stationary values, and we can therefore use a Taylor expansion for $r_{L}(\omega$, $\left.N_{1}\right) r_{R}\left(\omega, N_{2}\right)$ in (3):

$$
\begin{aligned}
r_{L}\left(\omega, N_{1}\right) r_{R}\left(\omega, N_{2}\right)-1 \\
\simeq-j\left(\tau_{\mathrm{in}}+\tau_{\mathrm{ex}}\right)\left(\omega-\omega_{s}\right) \\
\quad+\frac{\partial \ln r_{L}}{\partial N_{1}} \Delta N_{1}+\frac{\partial \ln r_{R}}{\partial N_{2}} \Delta N_{2}
\end{aligned}
$$

where $\Delta N_{i}=N_{i}-N_{i s}(i=1,2)$ and

$$
\begin{aligned}
\tau_{\text {in }} & =j \frac{\partial \ln r_{L}}{\partial \omega} \\
\tau_{\text {ex }} & =j \frac{\partial \ln r_{R}}{\partial \omega}=\frac{2 l_{2}}{c}\left(n_{2 g}+\Delta n_{2}\left(N_{2 s}\right)\right)
\end{aligned}
$$

are the effective round-trip times in the active and PC sections, respectively. $c$ is the light velocity in vacuum, and $n_{2 g}$ is the group refractive index in the PC section. We note that the round-trip times are generally complex, but with the given form of $r_{R}$ and $k_{2}, \tau_{\mathrm{ex}}$ becomes real (cf. Appendix A). The frequency range in which (5) is valid is determined by the range in which $r_{R}\left(\omega, N_{2}\right)$ can be approximated by its linear expansion, and the method described in this paper therefore applies to short (discrete or integrated) external cavities.

We now define the envelope function

$$
A^{+}(t)=\frac{1}{2 \pi} \int_{0}^{\infty} E^{+}(\omega) e^{j\left(\omega-\omega_{s}\right) t} d \omega
$$

for the right traveling wave at the reference plane [10]. From (3) and (5), the following simple rate equation for $A^{+}(t)$ can be derived:

$$
\begin{aligned}
\frac{d}{d t} \ln A^{+}(t)= & \frac{1}{\tau_{\text {in }}+\tau_{\text {ex }}}\left\{\frac{\partial \ln r_{L}}{\partial N_{1}} \Delta N_{1}(t)\right. \\
& \left.+\frac{\partial \ln r_{R}}{\partial N_{2}} \Delta N_{2}(t)\right\} .
\end{aligned}
$$

In order to introduce the photon number $I_{p}(t)$ and the phase $\varphi(t)$ of the electric field [i.e., the envelope function $A^{+}(t)$ ], we make use of the relation [10]

$$
A^{+}(t) \propto \sqrt{I_{p}(t)} e^{j \varphi(t)}
$$

and obtain the desired rate equation

$$
\begin{aligned}
\frac{1}{2} \frac{d}{d t} & \ln I_{p}(t)+j \frac{d \varphi(t)}{d t} \\
& =\frac{1}{\tau_{\text {in }}+\tau_{\text {ex }}}\left\{\frac{\partial \ln r_{L}}{\partial N_{1}} \Delta N_{1}(t)+\frac{\partial \ln r_{R}}{\partial N_{2}} \Delta N_{2}(t)\right\}
\end{aligned}
$$

for the photon number and phase, valid for a short external cavity. A similar equation for a laser with arbitrary external cavity length was given in $[10$, eq. (22)], but here modulation of the external cavity was not considered.

The rate equations for the carrier densities in the active and $\mathrm{PC}$ sections take the usual form:

$$
\begin{aligned}
& \frac{d}{d t} N_{1}(t)=\frac{I_{a}(t)}{e V_{1}}-R_{1}\left(N_{1}\right)-G\left(\omega_{s}, N_{1}\right) \frac{I_{p}(t)}{V_{1}} \\
& \frac{d}{d t} N_{2}(t)=\frac{I_{\mathrm{PC}}(t)}{e V_{2}}-R_{2}\left(N_{2}\right)
\end{aligned}
$$

where $e$ is the electron charge and $G\left(\omega_{s}, N_{1}\right)=v_{g} g\left(\omega_{s}\right.$, $N_{1}$ ) is the gain (per unit time) of the active section. $v_{g}$ is the group velocity, $g$ is the gain per unit length (see Ap- 
pendix $\mathrm{A}$ ), and $V_{1}, V_{2}$ and $I_{a}(t), I_{\mathrm{PC}}(t)$ are the volumes and injection currents for the active and PC sections, respectively. The spontaneous recombination rate per unit volume is represented as

$$
R_{i}\left(N_{i}\right)=a_{i} N_{i}+b_{i} N_{i}^{2}+c_{i} N_{i}^{3}, \quad i=1 \text { or } 2
$$

where $a_{i}, b_{i}$, and $c_{i}$ are constants. The recombination constant $c_{i}$ accounts for Auger recombination.

A small-signal analysis of (10), (11a), and (11b) is given in Appendix B. The result is the following set of modulation responses:

$$
\begin{aligned}
\delta P(\Omega)= & 2 P_{s}\left[H_{1}(\Omega) \frac{C_{1 r}}{e V_{1}} \delta I_{a}(\Omega)+H_{2}(\Omega) \frac{C_{2 r}}{e V_{2}} \delta I_{\mathrm{PC}}(\Omega)\right] \\
\delta f(\Omega)= & \frac{1}{2 \pi}\left[H_{3}(\Omega) \frac{C_{1 i}}{e V_{1}} \delta I_{a}(\Omega)+\left\{C_{1 i} H_{4}(\Omega)\right.\right. \\
& \left.\left.+C_{2 i} H_{5}(\Omega)\right\} \frac{1}{e V_{2}} \delta I_{\mathrm{PC}}(\Omega)\right] \\
\delta N_{1}(\Omega)= & {\left[H_{3}(\Omega) \frac{1}{e V_{1}} \delta I_{a}(\Omega)+H_{4}(\Omega) \frac{1}{e V_{2}} \delta I_{\mathrm{PC}}(\Omega)\right] } \\
\delta N_{2}(\Omega)= & H_{5}(\Omega) \frac{1}{e V_{2}} \delta I_{\mathrm{PC}}(\Omega)
\end{aligned}
$$

where $P$ denotes the output power from the left facet and $\Omega=2 \pi f_{m}$ is the angular modulation frequency. The $C$-factors and the transfer functions $H_{1}-H_{5}$ are defined in Appendix B. $\delta P(\Omega)$ and $\delta f(\Omega)$ represent the intensity modulation (IM) and FM responses for given forms of the two modulation currents $\delta I_{a}$ and $\delta I_{\mathrm{PC}}$.

As can be seen from (13a)-(13d), the modulation responses generally contain two contributions, one for each of the modulation currents, except for $\delta N_{2}(\Omega)$, which depends only on $\delta I_{\mathrm{PC}}(\Omega)$. The complex addition of the two carrier density terms in (10) may lead to interference effects in some frequency intervals, as illustrated in the next section. In particular, $(13 \mathrm{c})$ shows that $N_{1}(t)$ can be modulated via the PC current. This is a result of the photoncarrier interaction in the active section, which leads to the well-known relaxation resonance in a laser above threshold.

The response $\delta N_{2}(\Omega)$ has a low-pass character with a cutoff frequency of $1 /\left(2 \pi \tau_{s 2}\right)$ and a dc value which is proportional to $\tau_{s 2}$, with $\tau_{s 2}$ being the carrier lifetime in the PC section. This illustrates the role of the carrier lifetime in this type of tunable laser and the characteristic tradeoff between modulation speed and efficiency.

The IM and FM responses can be rewritten in the form

$$
\begin{aligned}
\delta P(\Omega)= & 2 P_{s} C_{\mathrm{l} r} H_{1}(\Omega)\left[\frac{1}{e V_{1}} \delta I_{a}(\Omega)\right. \\
& \left.+\frac{j \Omega+\left(1 / \tau_{R}\right)}{j \Omega+\left(1 / \tau_{s 2}\right)} \frac{C_{2 r}}{C_{1},} \frac{1}{e V_{2}} \delta I_{\mathrm{PC}}(\Omega)\right]
\end{aligned}
$$

$$
\begin{aligned}
\delta f(\Omega)= & \frac{\delta f(0)}{1+j \Omega \tau_{s 2}}+\frac{1}{2 \pi} C_{1 i} H_{3}(\Omega)\left[\frac{1}{e V_{1}} \delta I_{a}(\Omega)\right. \\
& \left.+\frac{j \Omega+\left(1 / \tau_{R}\right)}{j \Omega+\left(1 / \tau_{s 2}\right)} \frac{C_{2 r}}{C_{1 r}} \frac{1}{e V_{2}} \delta I_{\mathrm{PC}}(\Omega)\right]
\end{aligned}
$$

where $\delta f(0)$ is the FM response at dc [see Appendix B, eq. (B10b)] and $\tau_{R}$ is the relaxation time constant. This shows that if both currents are modulated simultaneously with a current splitting ratio of

$$
\frac{\delta I_{a}(\Omega)}{\delta I_{\mathrm{PC}}(\Omega)}=-\frac{j \Omega+\left(1 / \tau_{R}\right)}{j \Omega+\left(1 / \tau_{s 2}\right)} \frac{C_{2 r}}{C_{1 r}} \frac{V_{1}}{V_{2}},
$$

the intensity modulation will be completely suppressed, and at the same time, the resonance peak in the FM response will be canceled. If we ignore the complications of parasitics, the required current splitting can easily be realized by the use of resistors and capacitors. The resulting FM response has the same simple low-pass form as $\delta N_{2}$, and further equalization may be applied to both currents while maintaining the ratio (15) in order to extend the FM response to higher frequencies [13].

The expressions given so far apply to the phase-tunable DFB laser. For the case of the tunable DBR laser, $r_{L}$ becomes the effective reflectivity of the active Fabry-Perot section,

$$
r_{L}\left(\omega, N_{1}\right)=r_{1} \exp \left\{-j 2 k_{1}\left(\omega, N_{1}\right) l_{1}\right\},
$$

and the reflectivity $r_{R}\left(\omega, N_{2}, N_{3}\right)$ is given by (2) with $r_{2}$ replaced by the effective reflectivity $r_{\mathrm{DBR}}\left(\omega, N_{3}\right)$ of the DBR section as seen from the interface between the PC and DBR sections [8]. $N_{3}$ is the carrier density in the DBR section. The complex wave number of the DBR section can be represented in a way similar to that for the PC section, and the refractive index change will lead to a change of the Bragg frequency, which can be used to control the oscillation frequency. In (10), an extra term containing $\delta N_{3}(t)$ should be added, and in (11a) and (11b), an equation for $N_{3}$ must be added. The rest of the analysis proceeds in the same way, and the carrier densities in the passive sections still depend only on the injection current for the particular section. We have studied the modulation properties of this laser as well, and we obtained results similar to those presented in the next section.

\section{Results And Discussion}

The modulation properties of multisection lasers depend strongly on the bias conditions, and it is therefore very useful to have an overview of the static tuning characteristics before selecting the point of operation. Fig. 2 shows a calculated example of the static frequency tuning characteristics for a phase-tunable DFB laser [7]. In the calculations, we have used the laser parameters given in Table I. Four regions of continuous tuning separated by discrete mode jumps across the Bragg frequency are observed. The slope of the curve indicates the FM efficiency at $\mathrm{dc}$, as mentioned in Appendix B, and depending on whether the slope is positive or negative, the FM will be 


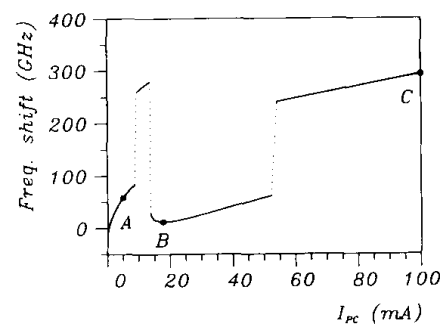

Fig. 2. Frequency shift versus $P C$ current. The points A. B, and C refer to the PC bias currents used in Fig. 3.

TABLE I

List of Parameter Valles

\begin{tabular}{|c|c|c|c|}
\hline Parameter & Symbol & Value & Unit \\
\hline Active section: & & & \\
\hline Length of active section & $l_{1}$ & 350 & $\mu \mathrm{m}$ \\
\hline Width of active region & $w$ & 0.8 & $\mu \mathrm{m}$ \\
\hline Thickness of active layer & $d$ & 0.18 & $\mu \mathrm{m}$ \\
\hline Refractive index & $n_{1}$ & 3.40 & \\
\hline Group refractive index & $n_{1 g}$ & 3.56 & \\
\hline Internal absorption & $\alpha_{1}$ & 21 & $\mathrm{~cm}^{-1}$ \\
\hline Recombination coefficient & $a_{1}$ & $1 \cdot 10^{8}$ & $\sec ^{-1}$ \\
\hline Recombination coefficient & $b_{1}$ & $8 \cdot 10^{-17}$ & $\mathrm{~m}^{3} / \mathrm{sec}$ \\
\hline Recombination coefficient & $c_{1}$ & $7.5 \cdot 10^{-41}$ & $\mathrm{~m}^{6} / \mathrm{sec}$ \\
\hline Confinement factor & $\Gamma_{1}$ & 0.15 & \\
\hline Gain curvature & $g_{\omega}$ & $0.73 \cdot 10^{-24}$ & $\mathrm{~m}^{-1} \sec ^{2}$ \\
\hline Gain coefficient & $g_{N}$ & $4.83 \cdot 10^{-21}$ & $\mathrm{~m}^{2}$ \\
\hline Carrier density at transparency & $N_{0}$ & $1.37 \cdot 10^{24}$ & $\mathrm{~m}^{-3}$ \\
\hline $\begin{array}{l}\text { Extrapolated wavelength of the } \\
\text { gain peak for } N_{1}=N_{0}\end{array}$ & $\lambda_{p}\left(N_{0}\right)$ & 1.60 & $\mu \mathrm{m}$ \\
\hline $\begin{array}{l}\text { Shift of gain peak with carrier } \\
\text { density }\end{array}$ & $d \omega_{p} / d N$ & $2.12 \cdot 10^{-11}$ & $\mathrm{~m}^{3} \mathrm{sec}^{-1}$ \\
\hline Facet reflectivity & $r_{1}^{2}$ & $32 \%$ & \\
\hline Linewidth enhancement factor & $\alpha$ & 6.6 & \\
\hline Reference wavelength & $\lambda_{\text {ref }}$ & 1.55 & $\mu m$ \\
\hline Reference carrier density & $N_{\text {ref } f}$ & $2 \cdot 10^{24}$ & $\mathrm{~m}^{-3}$ \\
\hline Phase control section: & & & \\
\hline Length of $\mathrm{PC}$ section & $I_{2}$ & 200 & $\mu \mathrm{m}$ \\
\hline Width of waveguide layer & & 1.2 & $\mu \mathrm{m}$ \\
\hline Thickness of waveguide & & 0.23 & $\mu \mathrm{m}$ \\
\hline Refractive index & $n_{2}$ & 3.4 & \\
\hline Group refractive index & $n_{2 g}$ & 3.4 & \\
\hline Internal absorption & $\alpha_{2}$ & 17 & $\mathrm{~cm}^{-1}$ \\
\hline Recombination coefficient & $a_{2}$ & $1 \cdot 10^{8}$ & $\sec ^{-1}$ \\
\hline Recombination coefficient & $b_{2}$ & $8 \cdot 10^{-17}$ & $\mathrm{~m}^{3} / \mathrm{sec}$ \\
\hline Recombination coefficient & $c_{2}$ & $4 \cdot 10^{-41}$ & $\mathrm{~m}^{6} / \mathrm{sec}$ \\
\hline Confinement factor & $\Gamma_{2}$ & 0.3 & \\
\hline $\begin{array}{l}\text { Index derivative with respect to } \\
\text { carrier density }\end{array}$ & $d n / d N$ & $-4.2 \cdot 10^{-27}$ & $m^{3}$ \\
\hline $\begin{array}{l}\text { Absorption derivative with respect } \\
\text { to carrier density }\end{array}$ & $d \alpha / d N$ & $1.8 \cdot 10^{-21}$ & $\mathrm{~m}^{2}$ \\
\hline Facet reflectivity & $r_{2}^{2}$ & $32 \%$ & \\
\hline
\end{tabular}

in phase or in counterphase with the PC current. Generally, the magnitude of the slope decreases for increasing injection level because Auger recombination limits the efficiency at high injection. On the other hand, the carrier lifetime decreases with increasing injection, which makes the FM response extend to higher frequencies.

This is clearly seen in Fig. 3(a) and (b), which shows the magnitude and phase of the FM response for the three different bias points $\mathrm{A}, \mathrm{B}$, and $\mathrm{C}$ in Fig. 2. At point $\mathrm{A}$ ( 5 $\mathrm{mA}$ ), the dc response and cutoff frequency are $8 \mathrm{GHz} / \mathrm{mA}$ and $190 \mathrm{MHz}$, respectively, and at point $\mathrm{C}(100 \mathrm{~mA})$, the corresponding values are $1.1 \mathrm{GHz} / \mathrm{mA}$ and $1.6 \mathrm{GHz}$. At point $\mathrm{B}(17.7 \mathrm{~mA})$, the static characteristic is near a minimum, and the FM efficiency at dc is very small. In the region above $1 \mathrm{GHz}$, a clear relaxation resonance peak (a)

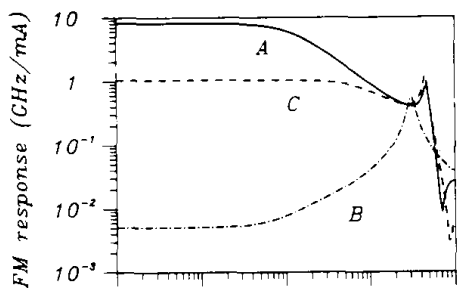

(b)

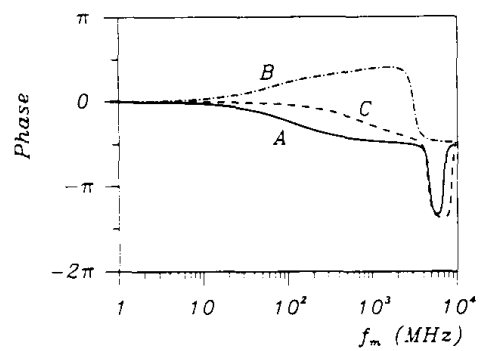

Fig. 3. FM response versus modulation frequency at three different $P C$ bias currents. (a) Magnitude. (b) Phase. The bias current $I_{a t .0}$ is $30 \mathrm{~mA}$. and the threshold currents at $\mathrm{A}, \mathrm{B}$, and $\mathrm{C}$ are $17.5,16.3$, and $21.8 \mathrm{~mA}$, respectively [7].

is observed, and intricate "interference" effects occur between the two FM contributions. The phase is 0 at low frequencies (if the static curve has a positive slope) and changes smoothly, except in the vicinity of the resonance peak. The presence of the resonance peak is a new result, which has not been reported experimentally. In the present analysis, we have not included the effect of nonlinear gain (gain compression), which could have some influence on the strength and shape of the resonance peak, but gain compression will not completely eliminate the resonance [14]. The resonance can, however, be shifted to higher frequencies by increasing the bias current to the active section. Below $1 \mathrm{GHz}$, our calculations are in good agreement with published experiments [3].

Fig. 4(a) and (b) shows the FM and IM responses when the active section is modulated (dotted curves) and when the PC section is modulated (dashed curves). As seen from the figure and predicted by (13a) and (13b), modulation of either of the currents will result in both IM and FM modulation, in all cases with a resonant part centered around the relaxation frequency. For many practical applications, however, it would be desirable to have a pure IM or a pure FM transmitter. For direct detection systems, especially those operating at high bit rates and/or long distances, the FM gives rise to a chirp-induced power penalty, and for coherent transmission systems based on FSK or PSK modulation, the IM is an unwanted effect. In multichannel systems using optical amplifiers, the IM will lead to crosstalk power penalties [15]. We notice that the IM resulting from modulation of the PC section is about an order of magnitude lower than that from the active section, but it still may not be negligible.

As mentioned in the previous section, it is possible to suppress both the IM and the resonant part of the FM response, if both sections are modulated according to (15). For the bias currents $I_{a .0}=30 \mathrm{~mA}$ and $I_{\mathrm{PC} .0}=100 \mathrm{~mA}$, 
(a)

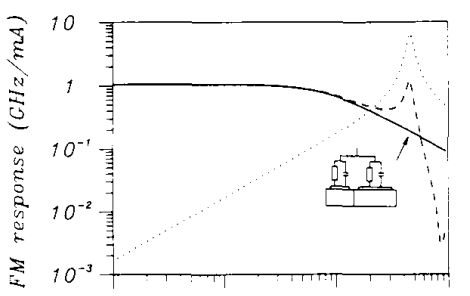

(b)

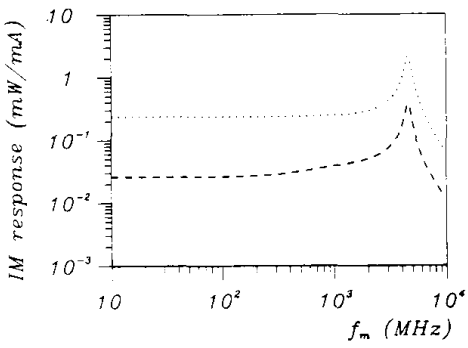

Fig. 4, Responses for three cases: modulation of the active section only (dotted curves), modulation of the PC section only (dashed curves), and simultaneous modulation of both sections with the splitting ratio (15) (solid curve). (a) FM. (b) IM. The inset in (a) shows the current splitting configuration. In the latter case, the modulation response is normalized by the current to the $\mathrm{PC}$ section. Bias currents: $I_{a, 0}=30 \mathrm{~mA}, I_{\mathrm{PC}, 0}=$ $100 \mathrm{~mA}$

the modulus and phase of the splitting ratio vary with frequency, as shown in Fig. 5. The major part of the current goes to the $\mathrm{PC}$ section, and at the resonance frequency $f_{R}$ $=4.6 \mathrm{GHz}$, the ratio is $\delta I_{a} / \delta I_{\mathrm{PC}}=0.18+j 0.012$. If we ignore the impedances of the laser sections, the splitting ratio can be realized by the simple circuit shown in the inlet of Fig. 4(a). The resistances $R_{a}, R_{\mathrm{PC}}$ and capacitances $C_{a}, C_{\mathrm{PC}}$ for the active and $\mathrm{PC}$ sections must satisfy the following conditions:

$$
\begin{aligned}
R_{u} C_{a} & =\tau_{R}=0.32 \mathrm{~ns} \\
R_{\mathrm{PC}} C_{\mathrm{PC}} & =\tau_{s 2}=0.19 \mathrm{~ns} \\
R_{a} / R_{\mathrm{PC}} & =-\frac{\tau_{R} V_{2} C_{1 r}}{\tau_{s 2} V_{1} C_{2 r}}=9.4 .
\end{aligned}
$$

The resulting FM response is also shown in Fig. 4(a) (solid curve). Below $1 \mathrm{GHz}$, the $\mathrm{FM}$ response is nearly unchanged by the current modulation of the active section due to the small splitting ratio and the low FM efficiency of the active section. Beyond $1 \mathrm{GHz}$, the resonant part has been eliminated, resulting in a very simple low-pass behavior, as predicted.

The present model only includes the effects on the FM response due to modulation of the carrier densities. As a consequence, the FM response due to modulation of the current to the active section approaches zero at low frequencies [cf. the dotted curve of Fig. 4(a) and eq. (B10b) of Appendix B]. At low frequencies, the dominant contribution comes from thermal effects, which may be included by adding a phenomenological term

$$
\delta f_{T}(\Omega)=-\frac{a_{T}}{1+j \Omega \tau_{T}} \delta I_{a}(\Omega)
$$

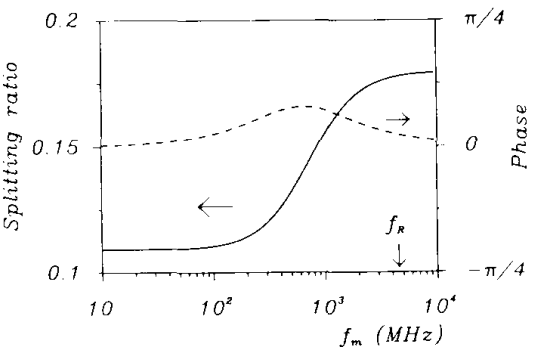

Fig. 5. Modulus and phase of optimum current splitting ratio (15).

to (14b). The dc efficiency $a_{T}$ is typically in the range of $0.1-1 \mathrm{GHz} / \mathrm{mA}$, and the thermal time constant $\tau_{T}$ is on the order of $0.1-1 \mu \mathrm{s}$. For these values, the term (17) will be small compared to $\delta f$ of (14b) because of the small splitting ratio [cf. Fig. 5].

\section{CONCluSion}

In this paper, a theoretical model has been presented for the modulation characteristics of tunable $D F B / D B R$ lasers with passive tuning sections. In the model, two effective reflectivities are used to characterize the active and passive sections. Numerical results have been presented for a phase-tunable DFB laser, but the model has also been applied to two- and three-section DBR lasers. It is shown that the FM characteristics depend critically on the operation point. In order to interpret a measured FM response, it is therefore important to have a measurement of the static frequency tuning characteristics. Generally, the FM efficiency decreases and the modulation bandwidth increases with increasing bias current to the PC section. Our calculations also show the presence of a resonance peak in the FM response when only the PC section is modulated. We have demonstrated that the intensity modulation can be completely suppressed by modulation of both the active and the PC sections with a simple splitting ratio between the currents. The same splitting ratio will eliminate the resonant part of the FM response and result in a low-pass response which is easy to equalize.

\section{APPENDIX A}

\section{The Complex Wave Numbers}

The effective reflectivities $r_{L}$ and $r_{R}$ are functions of the complex wave numbers in the active and passive sections. For the active section, the wave number is represented as

$$
k_{1}\left(\omega, N_{1}\right)=\frac{\omega n_{1}\left(\omega, N_{1}\right)}{c}+j \frac{1}{2}\left[g\left(\omega, N_{1}\right)-\alpha_{1}\right]
$$

where $n_{1}\left(\omega, N_{1}\right)$ is the refractive index, $g\left(\omega, N_{1}\right)$ is the modal gain, and $\alpha_{1}$ is the internal loss. The real part is expanded linearly around a reference point $\left(\omega_{\text {ref }}, N_{\text {ref }}\right)$, as explained in [10], and for the gain, we use a parabolic model, which includes the band-filling effect:

$$
\begin{gathered}
g\left(\omega, N_{1}\right)=g_{N}\left(N_{1}-N_{0}\right)-g_{\omega}\left(\omega-\omega_{p}\left(N_{1}\right)\right)^{2} \\
\omega_{p}\left(N_{1}\right)=\omega_{p}\left(N_{0}\right)+\frac{d \omega_{p}}{d N}\left(N_{1}-N_{0}\right) .
\end{gathered}
$$


Here, $\omega_{p}\left(N_{1}\right)$ is the angular optical frequency of the gain peak, $N_{0}$ is the carrier density at transparency, $g_{N}$ is the gain coefficient, $g_{\omega}$ is the gain curvature, and $\omega_{p}\left(N_{0}\right)$ is the (extrapolated) angular frequency of the gain peak for $N_{1}=N_{0}$. The effect of nonlinear gain is not included.

The wave number for the $\mathrm{PC}$ section is written as [7]

$$
\begin{aligned}
k_{2}\left(\omega, N_{2}\right)= & \frac{\omega}{c}\left[n_{2}(\omega)+\Delta n\left(N_{2}\right)\right] \\
& -j \frac{1}{2}\left[\alpha_{2}+\Delta \alpha_{2}\left(N_{2}\right)\right]
\end{aligned}
$$

where $n_{2}$ and $\alpha_{2}$ are the refractive index and the absorption per unit length without current injection and

$$
\begin{aligned}
& \Delta n_{2}\left(N_{2}\right)=\Gamma_{2} \frac{d n}{d N} N_{2} \\
& \Delta \alpha_{2}\left(N_{2}\right)=\Gamma_{2} \frac{d \alpha}{d N} N_{2}
\end{aligned}
$$

are the changes induced by current injection. $\Gamma_{2}$ is the confinement factor of the waveguide, and $d n / d N$ and $d \alpha / d N$ are coefficients describing the free-carrier plasma effect and the free-carrier absorption, respectively.

\section{APPENDIX B}

Small-Signal Analysis

From (10), (11a) and (11b), the small-signal FM and IM responses can be calculated. In the general case, both currents may be modulated:

$$
\begin{gathered}
I_{a}(t)=I_{a, 0}+\delta I_{a}(t), \\
I_{\mathrm{PC}}(t)=I_{\mathrm{PC} .0}+\delta I_{\mathrm{PC}}(t),
\end{gathered}
$$

and accordingly, the state variables are written as their stationary values plus a modulation term

$$
\begin{aligned}
I_{p}(t) & =I_{p . s}+\delta I_{p}(t), \quad \frac{d \varphi}{d t}=2 \pi \delta f(t) \\
N_{1}(t) & =N_{1 . s}+\delta N_{1}(t), \quad N_{2}(t)=N_{2 . s}+\delta N_{2}(t)
\end{aligned}
$$

where $\delta f(t)$ is the instantaneous frequency.

By elimination of the stationary values and subsequent Fourier transformation, the following set of equations is obtained:
Here, we have introduced the complex quantities

$$
\begin{aligned}
C_{1} & =\frac{1}{\tau_{\text {in }}+\tau_{\mathrm{ex}}} \frac{\partial \ln r_{L}}{\partial N_{1}}=C_{1 r}+j C_{1 i} \\
C_{2} & =\frac{1}{\tau_{\text {in }}+\tau_{\mathrm{ex}}} \frac{\partial \ln r_{R}}{\partial N_{2}}=C_{2 r}+j C_{2 i}
\end{aligned}
$$

and also $G_{s}=G\left(\omega_{s}, N_{1 s}\right)$. The parameters

$$
\tau_{R}=\left[\frac{d R_{1}}{d N_{1}}+v_{g} g_{N} \frac{I_{p, s}}{V_{1}}\right]^{-1} \text { and } \tau_{s 2}=\left[\frac{d R_{2}}{d N_{2}}\right]^{-1}
$$

are the relaxation time constant and the carrier lifetime in the PC section, respectively.

The system of equations can now be solved for each of the modulation terms. Instead of the modulation response for the photon number, we may introduce the modulation response $\delta P(\Omega)$ for the output power $P(t)$ from the left facet by noting that [10]

$$
P(t) \propto I_{p}(t),
$$

and hence,

$$
\frac{\delta I_{p}(\Omega)}{I_{p, s}}=\frac{\delta P(\Omega)}{P_{s}}
$$

where $P_{s}$ is the stationary value of $P(t)$. By introducing the transfer functions

$$
\begin{aligned}
& H_{1}(\Omega)=\frac{1}{-\Omega^{2}+j \Omega \frac{1}{\tau_{R}}+\omega_{R}^{2}} \\
& H_{2}(\Omega)=\frac{j \Omega+\left(1 / \tau_{R}\right)}{\left(j \Omega+\frac{1}{\tau_{s 2}}\right)\left(-\Omega^{2}+j \Omega \frac{1}{\tau_{R}}+\omega_{R}^{2}\right)} \\
& H_{3}(\Omega)=\frac{-\Omega^{2}+j \Omega \frac{1}{\tau_{R}}+\omega_{R}^{2}}{\left(j \Omega+\frac{1}{\tau_{s 2}}\right)\left(-\Omega^{2}+j \Omega \frac{1}{\tau_{R}}+\omega_{R}^{2}\right)} \frac{(\mathrm{B} 8}{C_{1 r}} \\
& \left.H_{4}(\Omega)=-\frac{\omega_{R}^{2}}{(j \Omega}\right)
\end{aligned}
$$

(B8d)

$$
\left[\begin{array}{lccc}
j \Omega & 0 & -2 I_{p, s} C_{1 r} & -2 I_{p, s} C_{2 r} \\
0 & 1 & -C_{1 i} & -C_{2 i} \\
G_{s} / V_{1} & 0 & j \Omega+\frac{1}{\tau_{R}} & 0 \\
0 & 0 & 0 & j \Omega+\frac{1}{\tau_{s 2}}
\end{array}\right]\left[\begin{array}{c}
\delta I_{p}(\Omega) \\
2 \pi \delta f(\Omega) \\
\delta N_{1}(\Omega) \\
\delta N_{2}(\Omega)
\end{array}\right]=\left[\begin{array}{c}
0 \\
0 \\
\frac{1}{e V_{1}} \delta I_{a}(\Omega) \\
\frac{1}{e V_{2}} \delta I_{\mathrm{PC}}(\Omega)
\end{array}\right]
$$




$$
H_{5}(\Omega)=\frac{1}{j \Omega+\frac{1}{\tau_{s 2}}},
$$

this leads to the expressions given in (13a)-(13d). Here, $\Omega=2 \pi f_{m}$ is the angular modulation frequency, and

$$
\omega_{R}^{2}=2 I_{p, s} C_{1 r} \frac{G_{s}}{V_{1}}=\left(2 \pi f_{R}\right)^{2}
$$

where $f_{R}$ is the relaxation resonance frequency.

The dc values of the modulation responses are given by

$$
\begin{aligned}
\delta P(0) & =2 P_{s}\left[\frac{C_{1 r}}{\omega_{R}^{2} e V_{1}} \delta I_{a}+\frac{\tau_{s 2} C_{2 r}}{\tau_{R} \omega_{R}^{2} e V_{2}} \delta I_{\mathrm{PC}}\right] \\
\delta f(0) & =\frac{1}{2 \pi}\left(C_{2 i}-\frac{C_{2 r}}{C_{1 r}} C_{1 i}\right) \frac{\tau_{s 2}}{e V_{2}} \delta I_{\mathrm{PC}} \\
\delta N_{1}(0) & =-\frac{C_{2 r}}{C_{1 r}} \frac{\tau_{s 2}}{e V_{2}} \delta I_{\mathrm{PC}} \\
\delta N_{2}(0) & =\frac{\tau_{s 2}}{e V_{2}} \delta I_{\mathrm{PC}}
\end{aligned}
$$

and are directly related to the slopes of the corresponding static tuning curves (cf. Fig. 2 and [7]).

\section{REFERENCES}

[1] Y. Yoshikuni and G. Motosugi, " Multielectrode distributed feedback laser for pure frequency modulation and chirping suppressed amplitude modulation," J. Lightwave Technol., vol. LT-5, pp. 516-522, Apr. 1987.

[2] H. Kawaguchi, K. Magari, H. Yasaka, M. Fukuda, and K. Oe, "Tunable optical-wavelength conversion using an optically triggerable multielectrode distributed feedback laser diode." IEEEJ. Quantum Electron., vol. QE-24, pp. 2153-2159, Nov. 1988.

[3] S. Murata, I. Mito, and K. Kobayashi. "Frequency modulation and spectral characteristics for a $1.5 \mu \mathrm{m}$ phase-tunable DFB laser," Electron. Lett., vol. 23, pp. 12-14, Jan. 1987.

[4] - "Over $720 \mathrm{GHz}(5.8 \mathrm{~nm})$ frequency tuning by a $1.5 \mu \mathrm{m}$ DBR laser with phase and Bragg wavelength control regions," Electron. Lett., vol. 23, pp. 403-405, Apr. 1987.

[5] Y. Kotaki, M. Matsuda, M. Yano, H. Ishikawa, and H. Imai, “1.55 $\mu \mathrm{m}$ wavelength tunable FBH-DBR laser," Electron. Lett., vol. 23, pp. 325-327, Mar. 1987

[6] T. L. Koch, U. Koren, and B. I. Miller, "High performance tunable $1.5 \mu \mathrm{m}$ InGaAs/InGaAsP multiple quantum well distributed Bragg reflector lasers," Appl. Phys. Lett., vol. 53, pp. 1036-1038, Sept. 1988

[7] H. Olesen, X. Pan, and B. Tromborg, "Theoretical analysis of tuning properties for a phase-tunable DFB laser, "IEEE J. Quantum Electron., vol. QE-24, pp. 2367-2375, Dec. 1988.

[8] X. Pan, H. Olesen, and B. Tromborg, "A theoretical model of multielectrode DBR lasers," IEEE J. Quantum Electron., vol. QE-24, pp. 2423-2432, Dec. 1988

[9] - .FM characteristics of multi-electrode DFB and DBR lasers," in Proc. IEEE Int. Semiconductor Laser Conf, Boston, MA. Aug. 1988, pp. 114-115

[10] B. Tromborg, H. Olesen, X. Pan, and S. Saito, "Transmission line description of optical feedback and injection locking for Fabry-Perot and DFB lasers," IEEE J. Quantum Electron., vol. QE-23, pp. 1875 1889, Nov. 1987.

[11] W. Streifer, R. D. Burnham, and D. R. Scifres, "Effect of external reflectors on longitudinal modes of distributed feedback lasers, "IEEE J. Quantum Electron., vol. QE-11, pp. 154-161, Apr. 1975.
[12] Y. Tohmori, F. Kano, M. Oishi, Y. Kondo, M. Nakao, and K. Oe, "Narrow linewidth and low chirping characteristics in high power operating butt-jointed DBR lasers grown by MOVPE," Electron. Lett., vol. 24, pp. 1481-1483, Nov. 1988.

[13] O. Nilsson, L. Gillner, and E. Goodbar, "Formulas for direct frequency modulation response of two-electrode diode lasers: Proposals for improvement," Electron. Lett., vol. 23, pp. 1371-1372. Dec. 1987.

[14] J. E. Bowers, T. L. Koch, B. R. Hemenway, D. P. Wilt, T. J. Bridges, and E. G. Burkhardt, "High-frequency modulation of 1.52 $\mu \mathrm{m}$ vapour-phase-transported InGaAsP lasers," Electron. Lett., vol. 21, pp. 297-299, Mar. 1985.

[15] M. G. Öberg, N. A. Olsson, and L. D. Tseng, "Crosstalk in a two channel coherent fiber optic FSK system using a semiconductor laser amplifier," J. Lightwave Technol., vol. LT-6, 1988.

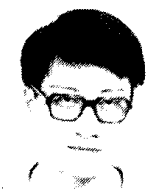

Xing Pan was born in Chongquing, China. on September 16, 1963. He received the B.S. degree in 1983 from the Chengdu Institute of Radio Engineering, Chengdu, China, and the M.Sc. degree in 1986 from the Technical University of Denmark, Lyngby, both in electrical engineering.

Currently, he is working towards the Ph.D. degree at the Technical University of Denmark. His research interests are in DFB lasers and optical feedback effects.

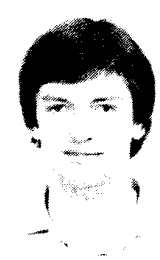

Henning Olesen was born in Vinderup. Denmark, on October 11. 1955. He received the M.Sc and $\mathrm{Ph} . \mathrm{D}$. degrees in electrical engineering from the Technical University of Denmark, Lyngby, in 1980 and 1982 , respectively.

From 1982 to 1984 he was a Postdoctoral Research Associate at the Electromagnetics Institute, Technical University of Denmark, where he worked on coherent optical transmission systems and, in particular, the coherence and FM characteristics of semiconductor lasers. In 1983 he stayed as a Visiting Scientist at Musashino ECL, Nippon Telegraph and Telephone Corporation, Tokyo, Japan. In 1984 he joined TFL Telecommunications Research Laboratory, Hørsholm, Denmark. He has written or coauthored more than 35 scientific journal and conference publications. His research is primarily concerned with modeling and characterization of semiconductor lasers for coherent systems, in particular, multielectrode DFB and DBR lasers and lasers with optical feedback.

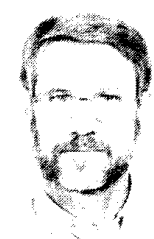

Bjarne Tromborg was born in 1940 in Denmark He received the M.Sc. degree in physics and mathematics from the Niels Bohr Institute, $\mathrm{Co}-$ penhagen, Denmark, in 1968

From 1968 to 1977 he was a Research Associate at NORDITA and the Niels Bohr Institute His research field was theoretical elementary particle physics, in particular, analytic $S$-matrix theory and electromagnetic corrections to hadron scattering. He coauthored a research monograph on dispersion theory. From 1977 to 1979 he taught at a high school. Since 1979 he has been with TFL Telecommunication. Research Laboratory, Hørsholm, Denmark, since 1987 as Head of the Optogroup. His present research interests include stability and noise properties of semiconductor lasers and quantum well structures in optoelectronic devices.

Mr. Tromborg received the Electroprize from the Danish Society of Engineers in 1981. 